\title{
Judgment stability and pseudorecognition of random and patterned configurations'
}

BRUCE M. ROSS

CENTER FOR RESEARCH IN THINKING AND LANGUAGE, CATHOLIC UNIVERSITY OF AMERICA

Thirty six simple patterns were judged as "random" or "patterned" by adult Ss. Category judgments were consistent whether Ss were told that $1 / 4,1 / 2$, or $3 / 4$ of the patterns were random or if no proportion was specified. In a second experiment, Ss viewed a series of slides previously judged patterned followed by a recognition series with two additional patterned slides inserted in a series of otherwise random slides. Although slides were never labeled random or patterned, Ss made the most false recognitions for the two patterned slides. In another condition where random and patterned slides were reversed, most false recognitions were made for the two random slides. Both Gamer's notion of hypothetical set size and an analysis of balanced elements fit the results, but the adequacy of both hypotheses can be questioned when applied to previous results with more complex patterns.

In a previous study (Ross \& Weiner, 1963) where Ss used patches of colored paper to construct squares that they considered "random," most of the obtained squares balanced their constituent elements around the presumed vertical and horizontal central axes of each square. It can be hypothesized that this result, which seems contrary to the common sense meaning of random, would be reversed if a square included only a small number of elements. One reason for this hypothesis is that in small squares balancing of elements occurs only if elements are exactly symmetrical; whereas in large squares, elements can be balanced without being exactly symmetrical. To test this hypothesis that small squares having balanced elements would not be considered random, an experiment was designed in which Ss judged whether squares with a minimal number of elements were either "random" or "patterned."

Interest also attaches to the fact that a square which is seldom designated random can, relatively at least, be considered a "good figure" among squares (patterns) possessing only a small number of elements. Garner (Garner, 1966; Garner \& Clement, 1963; Handel \& Garner, 1966) who has been interested in the problem of ranking figural goodness with patterns composed of a small number of elements, has proposed that the number of different patterns hypothetically obtainable (through pattern rotation and reflection) from a particular perceived pattern may be an important index of "goodness。" Specifically, a pattern that is a member of a set of patterns with potentially few different members will be judged to possess greater "goodness" than a pattern from a potentially large set. Despite the use of dichotomous forced judgments in the present experiment, the figural goodness of sets of patterns can be described by considering quantitative agreement among judgments. Thus it is possible to make a test of Garner's hypothesis.

In addition to concern with whether small random patterns are composed of balanced or unbalanced elements and the extent to which judgments are influenced by their membership in a hypothetical set of patterns, the first experiment had the purpose of determining the stability of judgments of randomness as a function of instructions. Conditions specified different proportions of randomness for the uniform array of patterns that each S viewed. Results could then be analyzed to determine if judgments maintained the same ranking despite make-believe proportionality differences given in the instructions.

A second experiment was concerned with whether the patterns characterized as random in the first experiment would be responded to differently from the other patterns if Ss received no suggestion that any patterns were random. The Ss were unaware of the purpose of this experiment in that they were led to believe that they were participating in a recognition memory experiment.

\section{Method}

\section{EXPERIMENT 1}

Stimuli. Each S saw a set of 36 patterms presented on $2 \times 2$ slides. Patterns were constructed by using all permutations of two black patches distributed on a 3 × 3 square grid. No grid lines were shown on the slides and there was no separation between black patches in those patterns where they were contiguous. A light border surrounded each square. As projected in various classrooms, the squares on the screen were approximately $2 \mathrm{ft}$. $\times 2 \mathrm{ft}$. with each dark patch 8 in. $x 8$ in.

Experimental design and procedure. There were four experimental conditions. In all conditions Ss saw the 36 slides in random order. At least two different orders were administered for each condition. For each pattern in the control condition Ss used answer sheets and encircled one of the entries: Patterned, Random, or ?. Ss were told only that some of the slides were patterned and some random and if they could not decide which they should encircle "?." This condition was administered in a group to two different classes.

In the three other conditions Ss were allowed only 


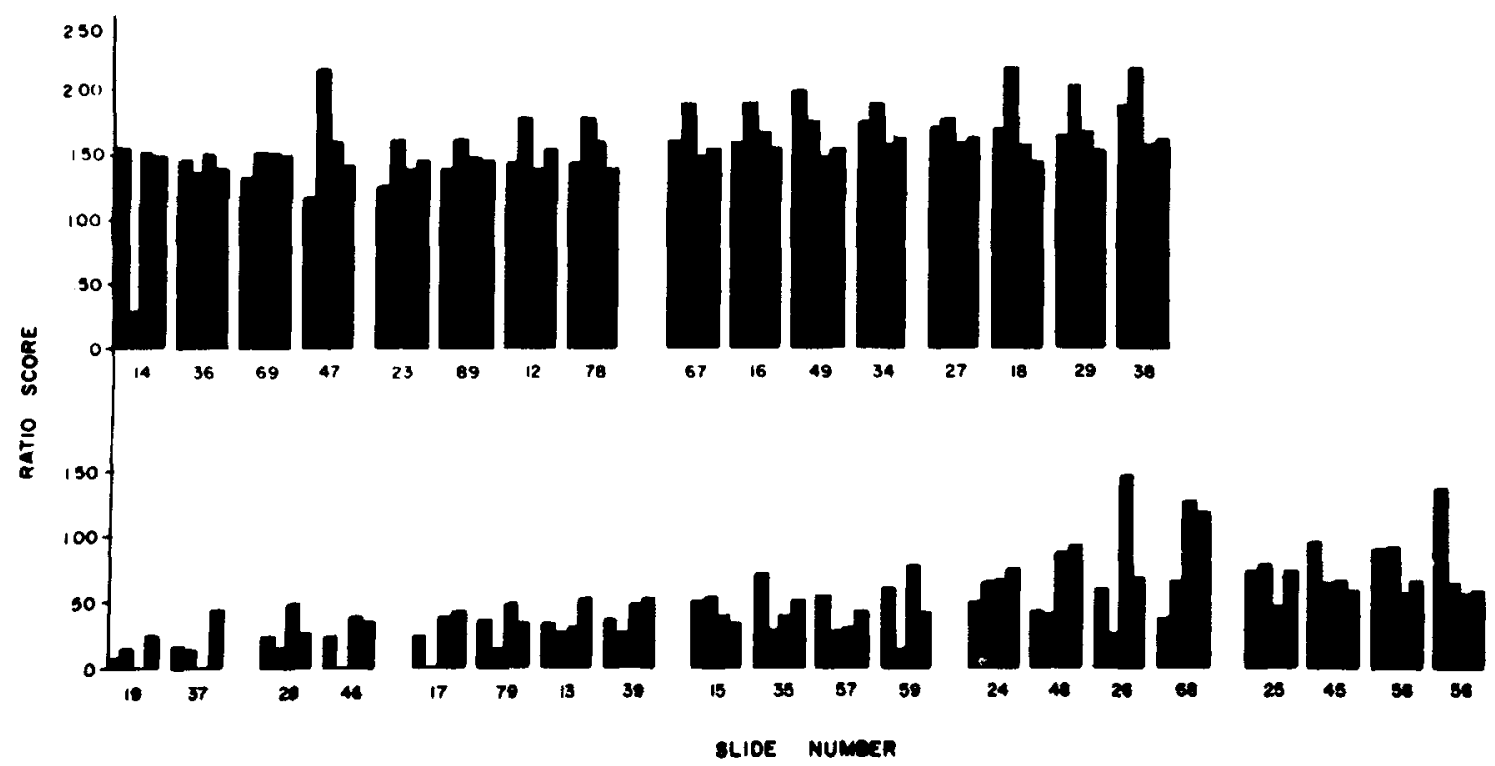

Fig. 1. Ratio scores of "randomness" for the 36 slide pattems with, left to right for each pattem, results for the control, $1 / 4,1 / 2$, and $3 / \%$ conditions.

two choices, either Patterned or Random. In these proportionality conditions Ss were told that either $1 / 4,1 / 2$, or $3 / 4$ of the slides had random patterns. For instance, for the $1 / 2$ condition instructions were: "Half of the slides you will see are random or chance black and white arrangements and half can be considered to be patterned. I am not going to tell you how many slides I will show you. Nevertheless, I want you to tell me for each slide individually whether you think it is a chance or patterned arrangement." As the procedure was administered individually in the three proportionality conditions, $S$ responded orally and $E$ recorded his judgment. In some conditions Ss were questioned after the experiment to elicit their criteria for judging. Vlewing time for each slide in all conditions was $10 \mathrm{sec}$, with $1 \mathrm{sec}$, between slides.

Subjects. In the control condition Ss were two adult education classes in psychology, both men and women, with an age range from 18 to about 60 . The three experimental groups were all composed of male undergraduate college students from Rutgers University.

\section{Results}

In Fig. 1 results are presented in terms of ratio scores of randomness on the ordinate with, left to right for each slide, the control, $1 / 4,1 / 2$, and $3 / 4$ conditions represented by bar height. Slides are named by designating the location of the two black patches on a particular slide. Numbering of the patches proceeds horizontally from left to right and top to bottom on a 9-place grid so that the patch in the upper left corner is designated 1 and that in the lower right corner 9. Individual ratio scores for each slide were obtained by dividing the number of "random" calls for that slide by the mean number of "random" calls per slide for the particular condition involved. The mean number of judgments of "random" given for all 36 slides in each condition is expressed on the ordinate as a score of 1.00 . Thus a score above 1.00 can be interpreted as a group judgment that the slide is random and a score below 1.00 that the slide is patterned.

It can be seen by inspection of Fig. 1 that, despite a few marked exceptions, there is good overall agreement across conditions. It can be concluded that judgments of randomness do possess considerable stability. These results also suggest that a composite score combining results from all four conditions might be the single best measure of judgments. Composite scores in which the means from the four conditions were combined to give a single mean ratio score for each slide are shown in Fig. 2.

The 20 slides shown in the bottom half of Fig. 2 all have values below 1.00 and the 16 slides in the top half of Fig. 2 all have values above 1.00. In consideration of this result that only 16 of the 36 slides $(44.4 \%)$ were consistently judged random, it seemed likely that Ss who were told $1 / 4$ of the slides were random had exceeded this proportion and those told $3 / 4$ of the slides were random had made a smaller number of "random" calls than requested. This proved to be the case as the mean number of "random" calls was $36.0 \%$ for the $1 / 4$ condition and $56.8 \%$ for the $3 / 4$ condition. In contrast, the number of "random" calls for the $1 / 2$ condition was close to the requested level at $49.7 \%$. As the control condition allowed a "?" response, it is not surprising that the mean number of "random" calls was fewer than for the other conditions, $25.0 \%$.

Results in Fig. 2 have been presented so that all 


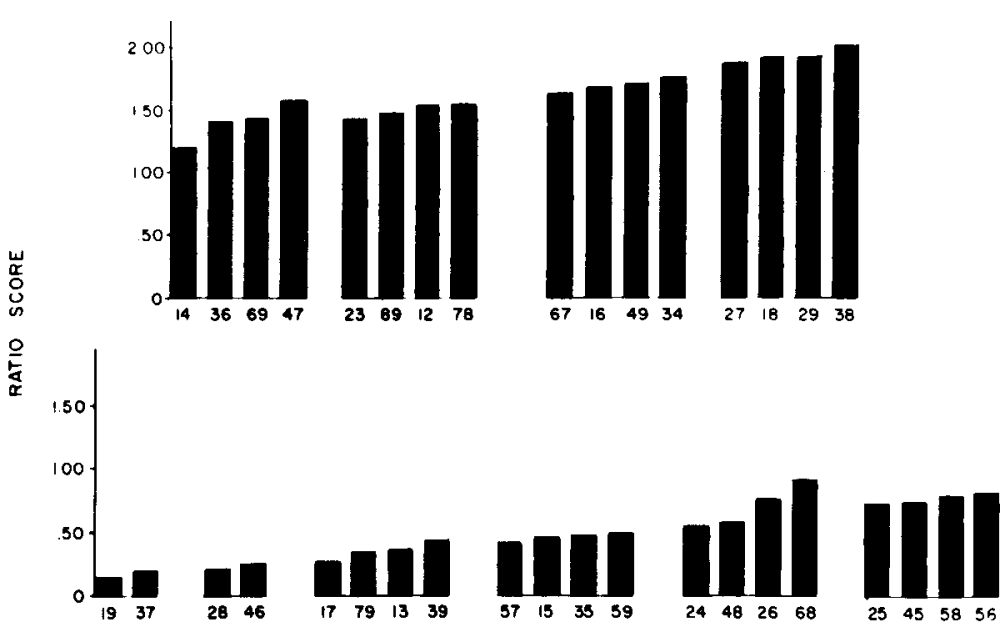

SLIDE NUMBER
Fig. 2. Composite ratio scores of "randomness" for the $\mathbf{3 6}$ slide patterns. slides that can duplicate each other either by rotation or reflection have been grouped together. Two conclusions can be drawn:

(1) All those slides in the upper half of Fig. 2 that qualify as random in that they are above a mean value of 1.00 are unbalanced in that the two black patches are asymmetrically placed around the horizontal, vertical, and two diagonal axes. It is necessary to consider diagonal axes because some of the patterned slides in the lower half of Fig. 2, notably Slides 19 and 37, are symmetrical only around the diagonal axes.

(2) Garner's hypothesis that figural goodness is a function of the size of the set of patterns that a particular pattern is drawn from is perfectly supported. The two sets obtained by rotation and reflection with only two members (lower left corner Fig. 2) produced the fewest "random" judgments, followed by the four sets with four members; while the two sets with eight members each in the upper half of Fig. 2 produced the most "random" 'judgments. Although belonging together in terms of rotation and reflection, the two sets of eight can each be subdivided into two subsets of four with regard to whether the two patches in each slide are mainly in the horizontal or the vertical plane. However, as can be observed in Fig. 2, results are the same for horizontal and vertical orientations within the same set. Classification solely in terms of the size of the set of which a pattern is a member appears to be an adequate index of figural goodness.

\section{EXPERIMENT 2}

This experiment assessed whether Ss would deal differently with slides called random as compared to those called patterned in Experiment 1 when no requirement to classify the slides was introduced. Instead of overt classification, contrast between random and patterned slides was introduced solely by context of presentation. The method used can be designated as pseudorecognition. Ss saw slides in an exposure series followed by a second series misleadingly designated as a recognition series, since all of the slides in the second series were different from those in the first series. Thus to be $100 \%$ correct Ss had to respond "no" to every slide in the second series. The experimental design was such that the extent to which differentiation among random and patterned slides took place could be detected by counting the number of "yes" responses of false recognition.

\section{Method}

Four conditions were administered in a classroom setting. In three conditions Ss first saw 18 of the 20 patterned slides in Fig. 2 projected in serial order. Ss were told only to "observe each slide carefully so that you can recognize it if you see it again." Each slide was exposed for $10 \mathrm{sec}$. with $1 \mathrm{sec}$. between slides. After a 30 sec. interval, Ss were shown serially the remaining 18 slides in Fig. 2 with the same time interval used with the exposure series; 16 slides had previously been judged random and two patterned in Experiment 1. For each slide $S$ wrote "yes" or "no" to indicate whether the viewed slide was among the 18 slides first shown. The three conditions varied principally as to which two patterned slides were inserted with the random slides in the recognition series and as to the ordinal positions at which these patterned slides were inserted. Presentation orders were also, of necessity, somewhat altered for each condition.

In Condition 1 , Slide 46 was in 6 th position and Slide 19 in 12 th position in the recognition series. It was presumed that these two slides, being among those most frequently judged patterned in Fig. 2, would encourage pseudorecognition to the greatest extent. In Condition 2, Slide 13 was in 8 th position and Slide 57 in 13th position in the recognition series. In Condition 3 , slide 45 was in 4 th position and Slide 68 in 14 th position in the recognition series. Note that 


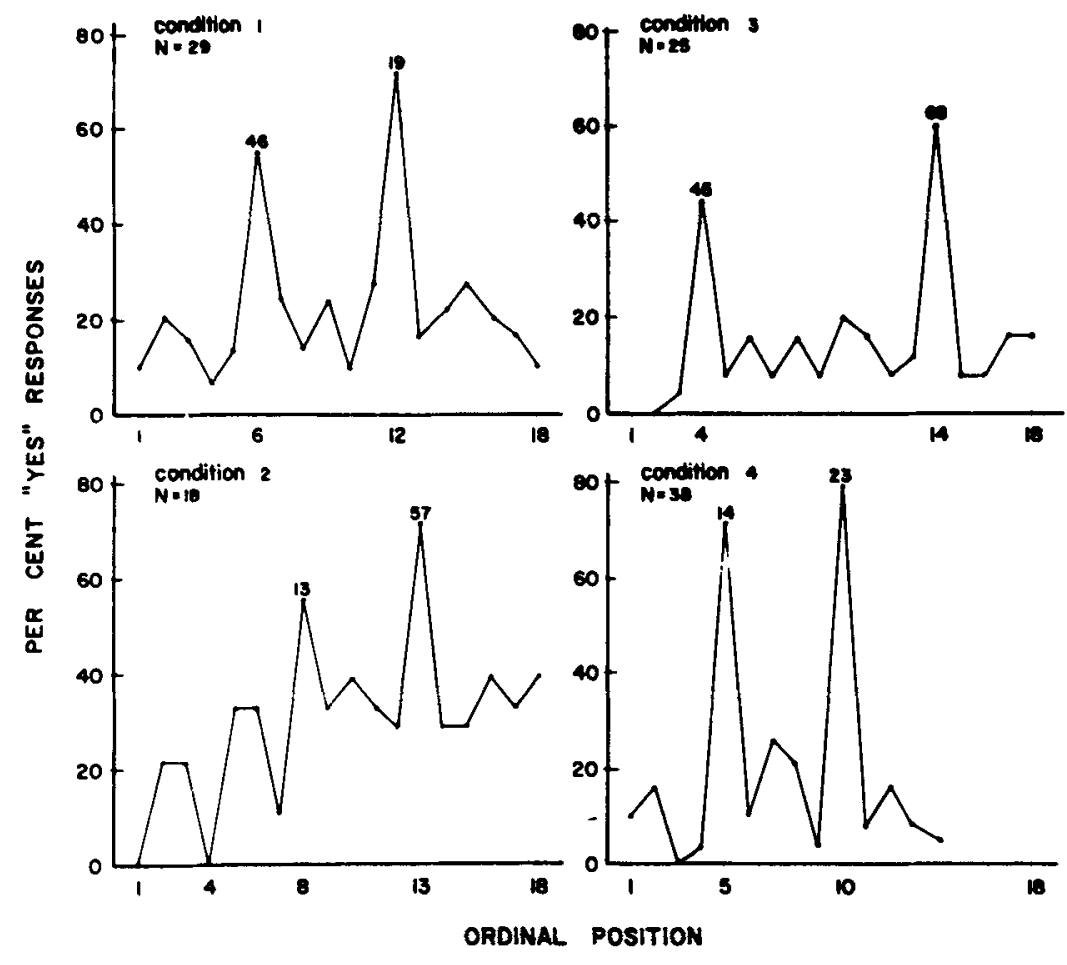

Fig. 3. Pseudorecognitions for the slides shown in the recognition series of Experiment 2.

Slides $13,57,45$, and 68 , although all had been judged patterned, were each from a different rotational set of four in Fig. 2.

Condition 4 was unlike Conditions 1,2 , and 3 in that only slides judged to be random were shown in the first series and two random slides were inserted among slides judged as patterned in the recognition series. Since, as shown in Fig. 2,20 slides were judged patterned and 16 random, both series were curtailed in Condition 4 to form a serles 14 slides in length. The two random slides in the recognition series were Slide 14 inserted in the 5th position and Slide 23 inserted in the 10th position. Both of these slides were from the same random set in Fig. 2, the set that showed slightly fewer random judgments than the other set also judged random. To keep both the first and recognition series of equal length, eight random slides were omitted. These omitted slides included those that were most frequently judged patterned; they were Slides $19,37,28,46,17,79,15$, and 35 . The administration procedure was the same as for the other three conditions.

Ss were undergraduate students. Condition 1 had an $\mathrm{N}$ of 29 from Rutgers University; Condition 2 had an $N$ of 18 from Rutgers University; Condition 3 had an $\mathrm{N}$ of 25 from Charles County (Md.) Community College; Condition 4 had an $\mathrm{N}$ of 38 from Catholic University.

\section{Results}

Figure 3 graphs results of the four conditions in terms of the percentage of false "yes" recognitions made for each condition. The results are unequivocal in that the two slides (named on each graph) that are out of place in each recognition series are also the two slides with the highest percentages of false recognitions. The contrast is perhaps slightly greater when the random slides are inserted among the patterned slides (Condition 4) and slightly less when the two unique patterned slides are close together in ordinal position (Condition 2). Nevertheless, it is clear for all conditions that the slides previously judged random and patterned are considered by Ss on the basis of their membership in these categories as well as on the basts of their specific configurations. Results of this experiment thus show that the distinguishing features that separate the "random" and "patterned" categories need neither be defined nor labeled to be effective.

\section{DISCUSSION}

In Experiment 1 it was found that Ss were relatively unaffected by instructions that different proportions of slides were random. This result indicates that Ss had some falrly marked criteria on which to base judgments, since many of the judgments in each condition had to be made before Ss could have formed an Idea of the total array of slides. Experiment 2 extends the results of Experiment 1 by showing that "random" and "patterned" are categories that are not just the result of arbitrary forced responding. When put in isolated context, a never-before-seen slide can be inferred as having the class membership given it in Experiment 1 despite a complete lack of any differential information or reinforcement.

The good agreement obtained between these judg- 
ments and Garner's (1966) notion that 'figural goodness" is inversely related to the size of the set of which a slide is a member indicates that set size may be the guiding criterion of judgment used by Ss. However, the extent to which elements are symmetrically balanced is also worth considering, as was noted by Garner and Clement (1963). Balancing of elements around diagonal as well as horizontal and vertical axes must be taken into account since some Ss reported that they used diagonal symmetry as a criterion for "patterned" responding. No Ss reported using set size as a criterion, so it could be that for this particular group of slides, set size is a purely formal restriction that happens to coincide with symmetry restrictions.

If diagonal symmetry is included among "patterned" criteria, the extent to which a slide is judged patterned can be directly related to whether the two black patches of each slide are symmetrically balanced around two, one, or zero axes. Thus, considering results in Fig. 2, Slides 19 and 37 are symmetrically balanced around two axes, viz., both diagonal axes. The other slides most frequently judged patterned, 28 and 46, are balanced around two axes, viz., the horizontal and vertical axes. The set 57, 15, 35, 59 and the set $24,48,26,68$ are each balanced around one diagonal axis only; while the set $17,39,45,56$ is balanced around the horizontal axis only, and the set $79,13,25,58$ around the vertical axis only. (Note that these latter two sets of four require a regrouping of the rotational sets of four shown together in Fig. 2.) Finally, the two sets of eight that produced a high frequency of "random" judgments do not have the two black patches balanced around any of the four axes.

One purpose of the present study was to determine if symmetrical balancing of elements with simplified configurations would tend to encourage "patterned" judgments. This tendency was found in contrast to previous results (Ross \& Weiner, 1963) where larger configurations with balanced elements were constructed as "random" patterns. It therefore appears that there is a point at which the size of a configuration, in terms of number of constituent elements, makes a difference. Thus with more complex configurations the notion of balancing of elements is not directly related to whether configurations are considered patterned. Note that configurations with balanced elements would necessarily have fewer rotations and reflections than other configurations. For this reason the set size of these preferred "random" configurations would be smaller than the set size of any unbalanced configurations. Thus smallness of set size also cannot be a good predictor of "patterned" for more complex configurations.

A reason why balanced elements might not be a criterion for "patterned" with more complex configurations was suggested in the introduction. With configurations larger than those used in this study, constituent elements can be balanced around horizontal, vertical, and diagonal axes and still not be exactly symmetrical. A reason why set size might not be an effective criterion for "patterned" with more complex configurations could be that with more elements figure-ground relations are established. Hence, it is possible that set size might remain an effective criterion of "goodness" if permutation sets for each perceived figure were tabulated separately, rather than the sets produced by rotation and reflection of the overall configurations. Whatever the validity of these speculations, the empirical finding remains that, as yet, a single descriptive rule does not account for judgments of "random" and "patterned" with configurations differing in complexity.

\section{References}

Gamer, W. R. To perceive is to know. Amer. Psychol., 1966, 21, 11-19.

Garner, W. R., \& Clement, D. E. Goodness of pattern and pattern uncertainty. J. verbal Learn. verbal Behav., 1963, 2, 446-452.

Handel, S., \& Gamer, W. R. The structure of visual pattern associates and pattern goodness. Percept. \& Psychophys., 1966, 1, 33-38.

Ross, B. M., \& Weiner, S. G. On making a random pattern. Percept. mot. Skills, 1963, 17, 587-600.

\section{Note}

1. This research was supported in part by Grant No. HD 02026 from the National Institute of Child Health and Human Development. I wish to acknowledge the advice and aid of Nissim Levy in devising the stimulus material.

(Accepted for publication March 6, 1967.) 